\title{
Single-particle levitation system for automated study of homogeneous solute nucleation
}

\author{
Adam P. Olsen, Richard C. Flagan, and Julia A. Kornfield \\ Division of Chemistry and Chemical Engineering, California Institute of Technology, \\ Pasadena, California 91125
}

(Received 3 April 2006; accepted 13 May 2006; published online 5 July 2006)

\begin{abstract}
We present an instrument that addresses two critical requirements for quantitative measurements of the homogeneous crystal nucleation rate in supersaturated aqueous solution. First, the need to perform repeated measurements of nucleation incubation times is met by automating experiments to enable programmable cycling of thermodynamic conditions. Second, the need for precise and robust control of the chemical potential in supersaturated aqueous solution is met by implementing a novel technique for regulating relative humidity. The apparatus levitates and weighs micron-sized samples in an electric field, providing access to highly supersaturated states. We report repeated observations of the crystal nucleation incubation time in a supersaturated aqueous sodium chloride droplet, from which we infer the nucleation rate. (C) 2006 American Institute of Physics.
\end{abstract}

[DOI: $10.1063 / 1.2212945$ ]

\section{INTRODUCTION}

Levitated, micron-sized particles are advantageous for studies of nucleation. Elimination of container surfaces that might catalyze nucleation and isolation of microscopic sample volumes allow access to highly supersaturated states. The large surface to volume ratio of small levitated samples enables rapid equilibration of heat and mass transport, and thereby minimizes the measurement time. Electrostatic forces provide a convenient means to levitate and weigh individual charged particles in an applied electric field. ${ }^{1}$ Electrostatic levitation has therefore found many applications in aerosol and materials research, including investigations of aqueous solution thermodynamics and crystal nucleation kinetics. ${ }^{1-12}$

Several researchers have recently employed electrostatic levitation to quantitatively investigate crystal nucleation kinetics in a variety of chemical systems. Nucleation is a stochastic process; when a supersaturated or supercooled state is created, nucleation occurs after a delay or incubation time. From repeated measurements of this incubation time in levitated droplets under similar conditions, a number of authors have inferred nucleation rates. Krämer et al. ${ }^{10}$ measured the nucleation rate of ice crystals in supercooled water by repeatedly injecting and trapping warm droplets into a cold balance; in a similar experiment Weidinger et al. ${ }^{12}$ measured homogeneous and heterogeneous crystal nucleation rates from $n$-alkane melts. Both studies detected crystallization through changes in optical properties. In studies of a nonvolatile solute in a volatile solvent, particle mass abruptly changes at the phase transition, providing a clear indicator of crystallization or dissolution. Supersaturated aqueous solution droplets maintain a metastable equilibrium at the water activity of the surrounding atmosphere until crystallization initiates spontaneous evaporation of water, ${ }^{2-5}$ the mass loss is readily detected as a change in the levitation voltage. Hamza et al. ${ }^{9}$ investigated the nucleation kinetics of potas- sium chloride in supersaturated aqueous solution by repeatedly injecting dilute droplets into a balance with fixed relative humidity. Knezic et al. ${ }^{8}$ measured the nucleation rate of a protein in aqueous droplets by cycling one particle between wet and dry states.

Crystal nucleation rates are extremely sensitive to thermal and chemical conditions. ${ }^{13}$ When, for example, Krämer et al. ${ }^{10}$ systematically varied the temperature, the measured ice crystal nucleation rate increased by a factor of 70 as the temperature was reduced by just $1 \mathrm{~K}$. Precise control of the thermodynamic state is thus critical to the measurement of nucleation rates. Only able to regulate the relative humidity (RH) within $\pm 0.5 \%$ RH during repeated experiments, Hamza et al. ${ }^{9}$ reported nucleation rates that varied by a factor of 300 . Since single particle nucleation studies require many observations to characterize each particular state, experimental reproducibility and efficiency are paramount. For aqueous solution experiments in particular, one must incorporate robust control systems for maintaining the desired relative humidity and temperature to ensure reproducibility.

Precise control of relative humidity in the vicinity of a levitated particle is required for investigations of aqueous solution thermodynamics and solute nucleation kinetics as highlighted above. Cohen $e t a l .^{4}$ slowly flowed a prescribed mixture of dry and saturated air streams through a large enclosure containing the levitation electrodes to minimize aerodynamic particle disturbance. Liang and $\mathrm{Chan}^{5}$ developed a quasiequilibrium technique that reduced the time required for experiments performed over a range of relative humidity. Using an instrument similar to that of Cohen et al., ${ }^{4}$ they continuously monitored particle mass during the transient buildup of relative humidity after a step change in the inlet flow. Tang and Munkelwitz, ${ }^{3}$ Richardson and Spann, ${ }^{2}$ and Knezic et $a l .{ }^{8}$ employed a vacuum technique in which the working gas was pure water vapor and relative humidity was determined by the total pressure. 
The aforementioned techniques all require that a particle be levitated and monitored for an extended time. Ideally, one would trap the particle in a stable potential well, however, according to Earnshaw's theorem, there are no local maxima or minima in a three-dimensional electrostatic field, only saddle points. A stable electrostatic levitation system cannot therefore be constructed; purely electrostatic levitation requires active control. Each of the studies highlighted above, in fact, utilized a stable variation of electrostatic levitation known as electrodynamic levitation. ${ }^{14,15}$ A conventional electrodynamic levitator balances charged particles against gravity with a vertical dc electric field and stabilizes them with an axisymmetric ac field. ${ }^{1}$ Many geometrical variations can successfully levitate particles; ${ }^{16}$ a particularly simple configuration is an ac ring electrode in the horizontal plane midway between two plates of a capacitor. A dc potential across the capacitor creates a vertical electric field for balancing gravitational forces. A potential applied to the ring creates a saddle point in the equipotential map of any vertical plane. When an ac potential of appropriate amplitude and frequency is applied to the ring, particles experience a time-averaged force towards the geometric null point. This dynamic stability allows the particle to be confined indefinitely to a finite volume of space. Unbalanced particles oscillate at the frequency of the ac potential. Vertical oscillations are eliminated only when the vertical dc electric field is adjusted to exactly balance gravity. Stray lateral fields can cause horizontal oscillations as well; several researchers have eliminated these by adding compensating lateral dc fields. ${ }^{17,18}$ Zheng et al. ${ }^{19}$ introduced an elegant design in which vertical and horizontal dc biases could be applied to a segmented ac ring electrode for three-dimensional compensation.

The electrodynamic levitator naturally lends itself to being controlled. The particle's position can be determined optically, and automated feedback control can be implemented to maintain the proper balancing potential. ${ }^{20,21}$ The de potentials required to balance a particle are proportional to external forces, so the vertical dc balancing potential provides a record of particle mass. The controller must be precisely aligned with the null point of the ac field to minimize particle oscillations, however, and any mechanical or electronic drift will reduce the accuracy of the mass measurement. Electrodynamic levitators with lateral compensation or purely electrostatic levitators may be integrated with three-dimensional position controllers to enable long term particle confinement ${ }^{18,22}$ these systems enable a more robust mass measurement since they do not require precise alignment with the dynamic null point.

This article describes a system for gravimetric analysis of nucleation from solution. The instrument employs an electrostatic particle levitator with a three-dimensional position controller; the levitator can also be operated as an electrodynamic balance by coupling the horizontal compensation electrodes to an ac potential. The instrument also incorporates strict temperature regulation and automated relative humidity control that is based on a novel continuous flow technique. The humidity controller is programmable, enabling exposure to any desired history, and experiments are fully automated for superior efficiency and reproducibility. Accuracy of rela-

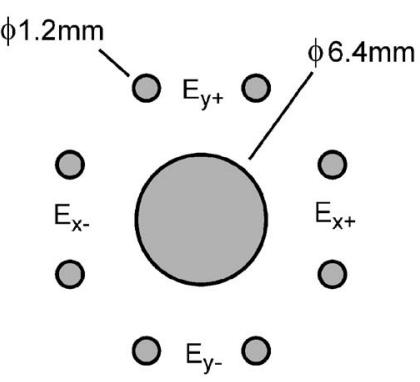

(a)

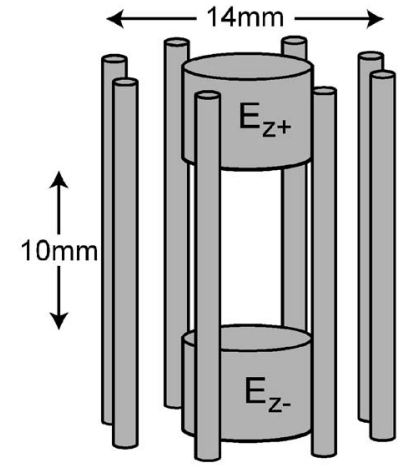

(b)
FIG. 1. Schematic of the electrode configuration designed for electrostatic and electrodynamic particle levitations showing (a) the top view and (b) a 3D representation. Each of the six isolated electrodes are labeled.

tive humidity control and mass measurement was verified by comparing the measured thermodynamics of aqueous sodium chloride solution droplets with a reported correlation. The instrument is programed to accumulate statistics of nucleation incubation times; nucleation statistics and inferred rates for a supersaturated sodium chloride droplet are reported.

\section{APPARATUS}

The particle levitation instrument is assembled from machined components, custom electronics, and commercially available devices. All electronic components are integrated with a personal computer (PC) equipped with data and image acquisition boards, enabling experiments that are automated and thus highly reproducible. The user interface is programed in LABVIEW.

\section{A. Levitation electrodes and environmental chamber}

The electrode configuration depicted in Fig. 1 consists of two dc disk electrodes $\left(E_{z+}\right.$ and $\left.E_{z-}\right)$ located above and below the particle and a coaxial cage of eight rod electrodes arranged on a regular octagon. Horizontal electrostatic forces can be generated by application of a symmetric dc bias between opposing pairs of rods (Table I). The geometry closely resembles that employed by Schlemmer et al. ${ }^{23}$ although the symmetry axis in that work was horizontal. The electrodes allow three-dimensional electrostatic force compensation so

TABLE I. Time dependent electric potentials applied to each of the six levitation electrodes and operating voltage limits.

\begin{tabular}{cc}
\hline \hline Electrode & Potential \\
\hline$E_{x+}$ & $V_{x}+V_{\mathrm{ac}} \cos \omega t$ \\
$E_{x-}$ & $-V_{x}+V_{\mathrm{ac}} \cos \omega t$ \\
$E_{y+}$ & $V_{y}+V_{\mathrm{ac}} \cos \omega t$ \\
$E_{y-}$ & $-V_{y}+V_{\mathrm{ac}} \cos \omega t$ \\
$E_{z+}$ & $V_{z}$ \\
$E_{z-}$ & $-V_{z}$ \\
& $V_{\mathrm{ac}}<2000 \mathrm{~V}$ at $30-300 \mathrm{~Hz}$ \\
& $\left|V_{x}\right|<15 \mathrm{~V}$ \\
& $\left|V_{y}\right|<15 \mathrm{~V}$ \\
& $\left|V_{z}\right|<150 \mathrm{~V}$ \\
\hline
\end{tabular}




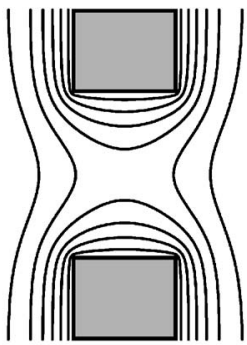

(a) $V_{x}=V_{y}=V_{z}=0$

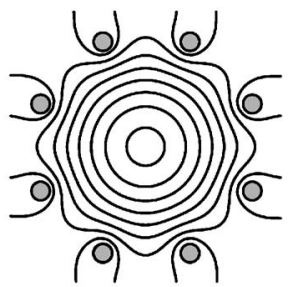

(c) $V_{x}=V_{y}=V_{z}=0$

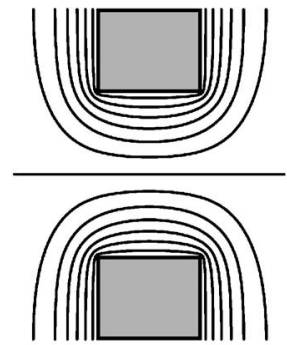

(b) $V_{x}=V_{y}=V_{a c}=0$

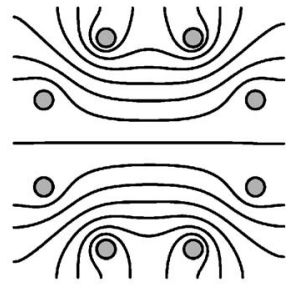

(d) $V_{x}=V_{z}=V_{a c}=0$
FIG. 2. Calculated equipotential contours in the $x=0$ plane for (a) the ac field and (b) the $z$ dc field, and in the $z=0$ plane for (c) the ac field and (d) the $y \mathrm{dc}$ field using the ion optics simulation software simION. Since Laplace's equation is linear, the total electric field is the sum of all component fields.

that a particle can be trapped indefinitely with proper feedback control, while allowing optical access along four coplanar axes.

This levitator can also be operated as an electrodynamic balance by biasing all of the cage electrodes with an ac potential. In practice, electrodynamic mode is used to capture a particle; $V_{\mathrm{ac}}$ is then decreased to zero during all experiments. The ac electric field generated by this electrode configuration [Figs. 2(a) and 2(c)] is highly axisymmetric in the vicinity of the particle even though the ac electrode is a discrete cage. The ac saddle point evident in Fig. 2(a) is responsible for electrodynamic focusing. The axial [Fig. 2(b)] and lateral [Fig. 2(d)] components of the dc electric field enable electrostatic balancing against gravitational, aerodynamic, and Brownian forces.

The electrodes are housed inside a machined stainless steel chamber that enables strict control of the particle's environment. The chamber body has an octagonal perimeter and a cylindrical internal cavity with an internal volume of approximately $40 \mathrm{cc}$ (Fig. 3). Eight view ports permit optical access to the particle between adjacent rod electrodes. The view ports provide $0.17 \mathrm{sr}\left(24^{\circ}\right)$ of access for laser illumination, particle imaging, and collection of angular light scattering. The body has annular channels machined into the sidewall above and below the plane of the view ports through which a heat transfer fluid can be circulated to attain precise temperature control (Fig. 4). Stainless steel end caps seal the chamber body from above and below. The chamber temperature is continuously monitored by two thermistors, one mounted on each end cap. An annular stainless steel frit (Mott Corporation) secured above an annular channel in the internal face of each end cap defines a balanced upward gas flow through the chamber. The resulting flow pattern enables rapid turnover of internal gas composition, while minimizing

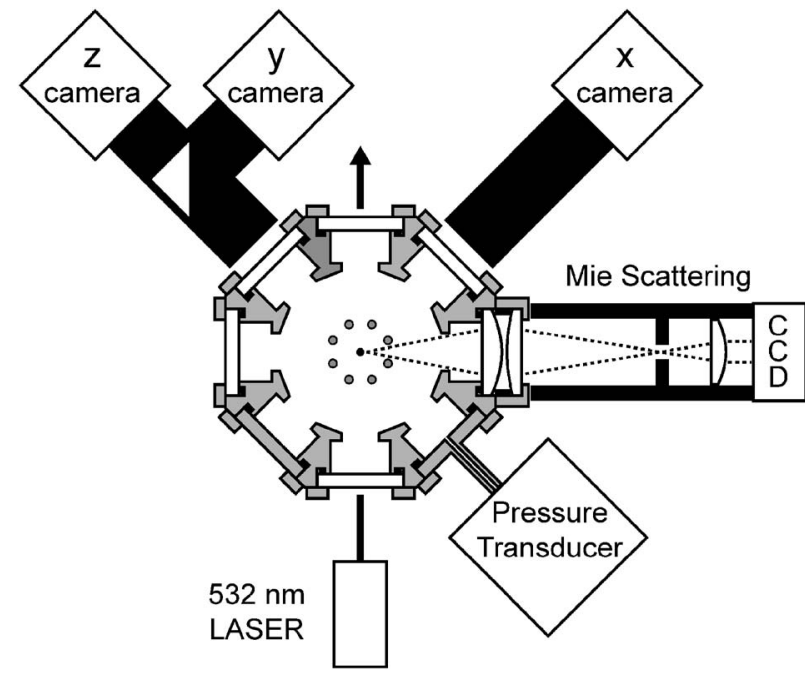

FIG. 3. Sectional view of the levitation chamber in the $z=0$ plane and peripheral components. Three linescan cameras detect particle displacement in $x, y$, and $z$ directions as indicated.

the aerodynamic drag force on the particle. The electrode assembly is secured to and electrically isolated from the end caps via ceramic feedthroughs. All joining surfaces are sealed with Viton O-rings.

A hole though the center of the bottom dc electrode facilitates capture of solid particles from a powdered sample according to the following procedure. An ensemble of particles is introduced to the interior of the chamber on the tip of a dielectric rod, and with the ac field adjusted safely below
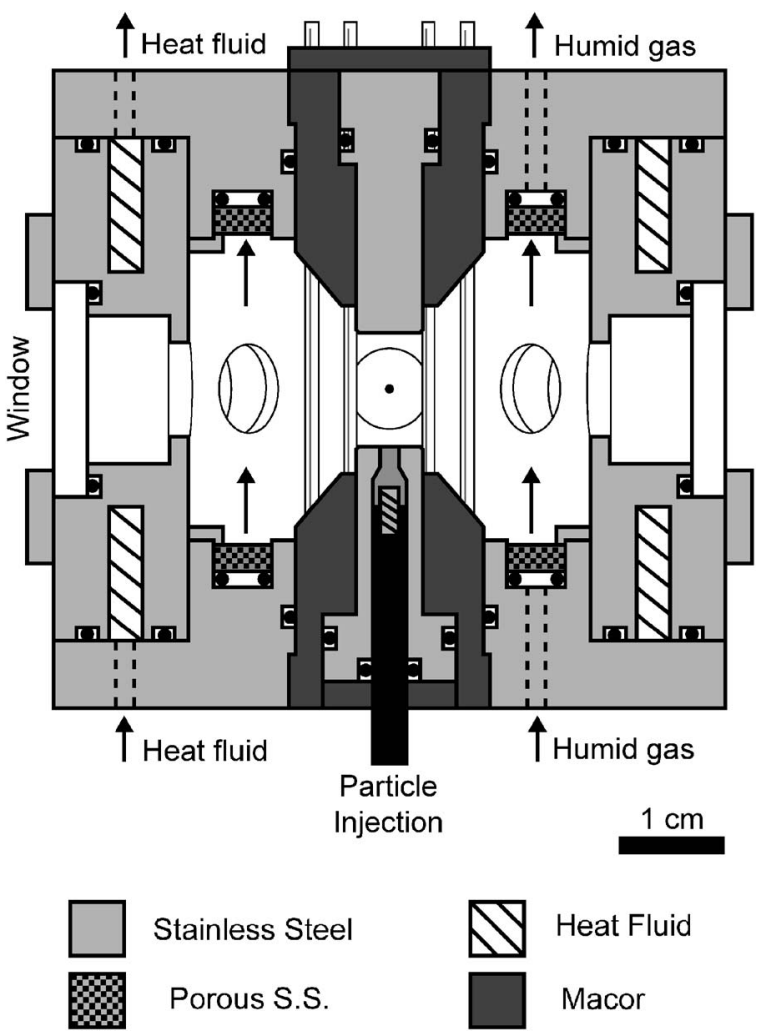

FIG. 4. Sectional view of the levitation chamber in the plane of the laser beam. Rod electrodes are positioned out of this plane and the plane of all optical axes as shown in Fig. 3. 
breakdown level, a mechanical pulse launches one or more particles into stable oscillation. A target particle is then isolated by appropriate adjustment of the dc potentials and reduction of the ac potential. The access hole is plugged and sealed during the experiment by a Delrin rod capped with stainless steel (Fig. 4).

\section{B. Particle imaging and position control}

Three-dimensional imaging and position control confine a particle within a finite viewing volume and provide a continuous record of particle mass. The levitated particle is illuminated by a linearly polarized $25 \mathrm{~mW} 532 \mathrm{~nm}$ diode laser beam (Melles Griot) (Fig. 3). Microscope tubes image the particle onto each of three mutually orthogonal analog charge-coupled device (CCD) linescan cameras (Perkin Elmer; LC3000). A beam splitter in one of the optical paths enables two orthogonal axes $(y$ and $z$ ) to be imaged through a single view port. A cylindrical lens in each optical path generates a line image perpendicular to the detector array. These $x, y$, and $z$ images are digitized by a multichannel frame grabber (Bitflow, Inc.; Raven). A digital proportional +integral+derivative (PID) algorithm generates $x, y$, and $z$ $\mathrm{dc}$ control voltages to maintain the particle at the intersection of both imaging axes and the laser beam. The controller operates at $40 \mathrm{~Hz}$ and is sufficient to stably confine particles in electrostatic mode without dynamic focusing.

The PID parameters are empirically determined for optimum performance. With proper tuning, the position controller is sufficiently sensitive to hold a levitated particle during the most rapid transients in mass that are anticipated for a given experiment without introducing excessive fluctuations at steady state. If the mass changes so rapidly that the controller cannot maintain the particle within the viewing volume, the control software is programed to briefly activate the ac field until the particle image is reacquired. This failsafe mechanism is occasionally needed after a nucleation event.

\section{Relative humidity control}

A novel technique for controlling the relative humidity inside the levitation chamber enables rapid and stable control of the concentration of levitated aqueous solution droplets. A constant mass flow rate [ca. 75 SCCM (standard cubic centimeter per minute at STP)] of gas through the chamber is maintained by simply placing a limiting orifice (O'Keefe Controls; $10 \mu \mathrm{m}$ ) ahead of a diaphragm vacuum pump as shown in the upper right corner of Fig. 5. An environmental control module regulates the total pressure inside the levitation chamber as it is continuously purged by a nitrogen stream that has been humidified to a fixed high relative humidity. The relative humidity inside the chamber is controlled by adjusting the pressure. A Nafion membrane shelland-tube humidifier (Perma Pure Inc.) generates a near saturated flow of humid nitrogen (ca. $10^{3}$ SCCM) and a high performance humidity sensor (Vaisala; HMP230) continuously monitors the humidity at ambient pressure $\left(p_{o}\right)$. The humidifier and relative humidity probe are maintained at the same temperature as the levitation chamber with circulating

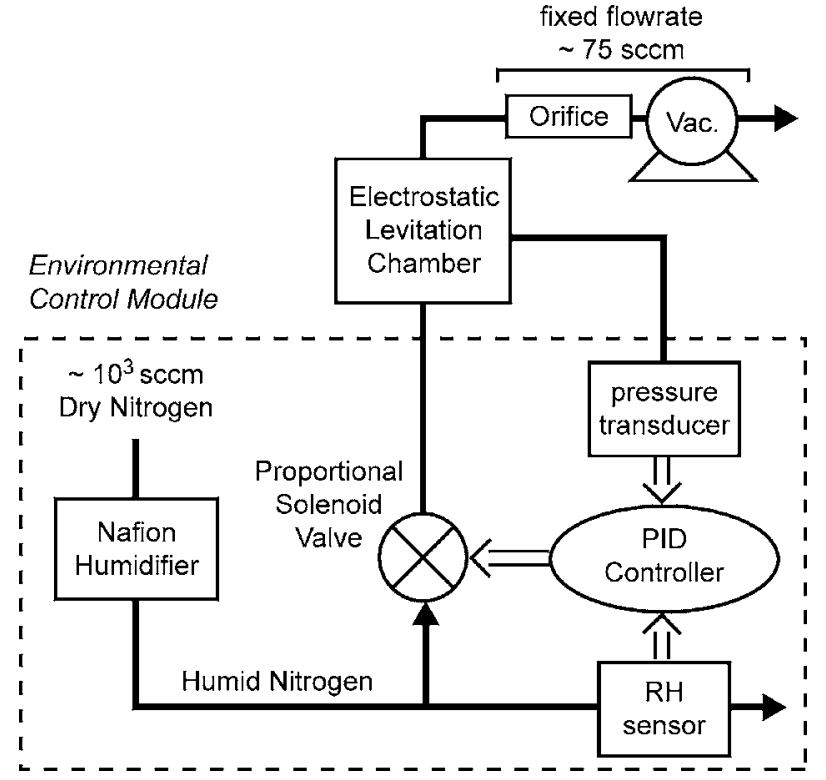

FIG. 5. Schematic of the environmental control module.

heat transfer fluid for stability. The recirculation bath (Neslab; RTE 7) has both heating and cooling capacities and is filled with a mixture of water and ethylene glycol for temperature control between -25 and $125 \pm 0.01{ }^{\circ} \mathrm{C}$. A proportional solenoid valve (Pneutronics; Lone Wolf) located just upstream from the levitation chamber serves as a variable pressure drop flow element for adjusting the total pressure inside the chamber. A transducer (MKS Instruments, Inc.; 222B) installed in one of the chamber access ports continuously monitors the chamber pressure $(p)$, which is digitized along with the output of the relative humidity sensor $\left(\mathrm{RH}_{o}\right)$. The relative humidity at the particle $\left(\mathrm{RH}_{p}\right)$ is then $\mathrm{RH}_{p}$ $=\left(p / p_{o}\right) \mathrm{RH}_{o}$. A digital PID control algorithm generates a control voltage for the proportional solenoid valve to keep $\mathrm{RH}_{p}$ at the specified value inside the levitation chamber. The controller operates at $40 \mathrm{~Hz}$ and can be programed to expose a levitated particle to any desired relative humidity history. In practice this technique enables stable control of relative humidity up to $95 \% \mathrm{RH}$.

\section{Collection of angular light scattering}

Angularly resolved scattered light from a levitated particle is collected through one of the view ports orthogonal to the illuminating laser beam (Fig. 4). The optical arrangement is similar to that reported by Krämer et al. ${ }^{10} \mathrm{~A}$ collimating lens fastened directly to the chamber in place of a window minimizes Fabry-Pérot interference that would result from passing the spherical wave front through the two parallel flat surfaces of a window. A second lens focuses the scattered light through an aperture (approximately $1.5 \mathrm{~mm}$ ) to filter off-axis radiation and a third lens recollimates the light onto the CCD detector of a video camera. The resulting unfocused particle image maps scattered light intensity as a function of scattering angle between approximately $78^{\circ}$ and $102^{\circ}$ relative to the illuminating laser beam. The images can be used to determine the size of liquid droplets by comparison with the Mie theory. ${ }^{24}$ Upon formation of heterogeneities in the 


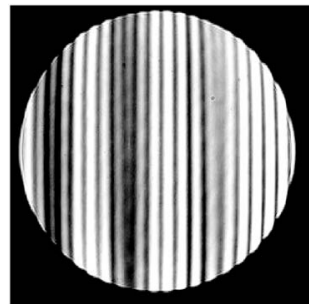

(a) $\mathrm{m} / \mathrm{m}_{\mathrm{o}}=3.0$ $D_{p}=30$ microns

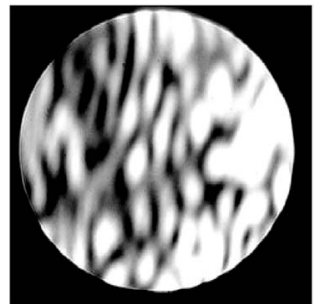

(b) $\mathrm{m} / \mathrm{m}_{\mathrm{o}}=1.0$
FIG. 6. Light scattering images of (a) an aqueous sodium chloride droplet and (b) a dry sodium chloride particle. Each image resolves light scattered between $78^{\circ}$ and $102^{\circ}$ relative to the incident laser beam.

supersaturated liquid, deviation from Mie scattering is observed. Conversely, when heterogeneities dissolve away, the Mie pattern reemerges. Thus, the images provide direct evidence of crystal nucleation and dissolution.

\section{RESULTS}

\section{A. Instrument characterization}

Angularly resolved light scattering provides an estimate of absolute sample size and can be used to differentiate liquid droplets and solid particles. The scattering image of the aqueous sodium chloride droplet reveals vertical fringes characteristic of an isotropic sphere [Fig. 6(a)]; the fringe spacing indicates a diameter of $30 \pm 1 \mu \mathrm{m}$ from comparison with Mie theory. The scattering image of a sodium chloride particle in the dry crystalline state is uncorrelated, indicating optical heterogeneity [Fig. 6(b)].

The long term stability of the instrument's humidity control was demonstrated by holding an aqueous sodium chloride droplet at $75 \% \mathrm{RH}$ and $21^{\circ} \mathrm{C}$ for $8 \mathrm{~h}$ (Fig. 7). The recorded mass has been normalized by the $8 \mathrm{~h}$ average and

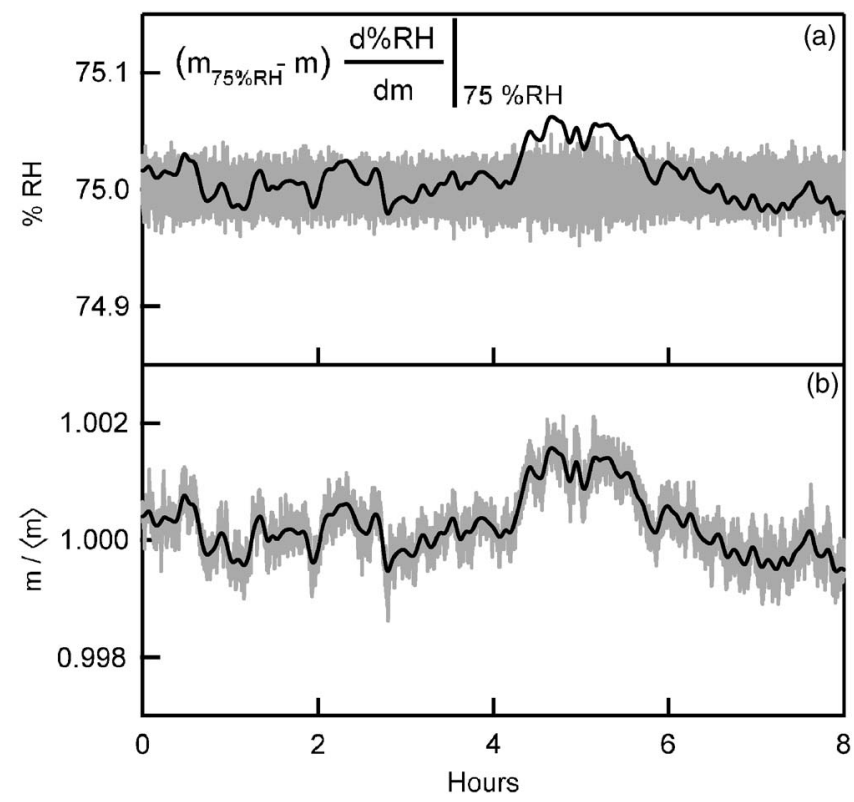

FIG. 7. Stability of an aqueous sodium chloride droplet at constant relative humidity. Particle mass is normalized by the $8 \mathrm{~h}$ average. Gray traces: Raw data collected at $30 \mathrm{~s}$ intervals. Black traces: (a) calculated $\mathrm{RH}$ and (b) filtered droplet mass. $T=21^{\circ} \mathrm{C}$.

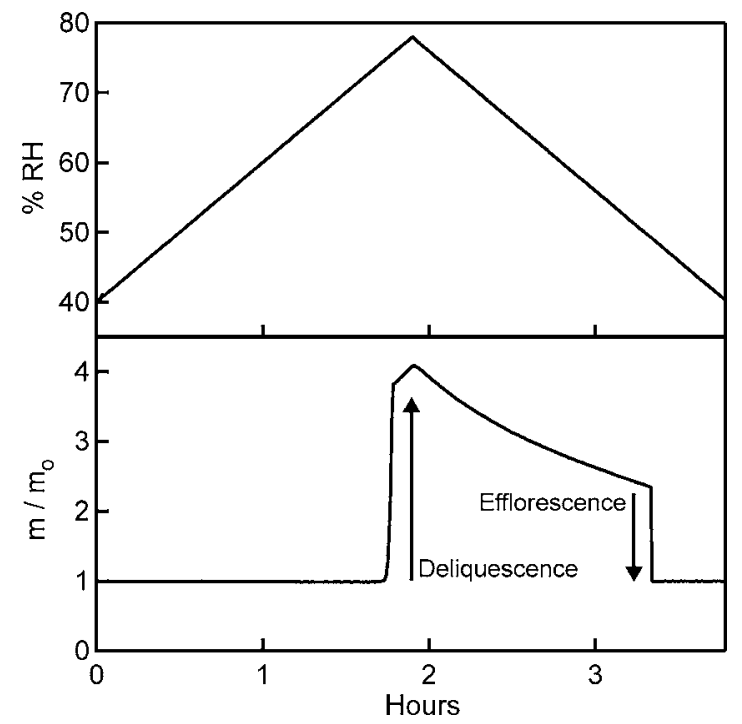

FIG. 8. Response of a sodium chloride particle to a continuous triangular relative humidity scan at $20 \% \mathrm{RH} / \mathrm{h}$. Particle mass was normalized by the mass of the dry state. Data points are recorded at $30 \mathrm{~s}$ intervals. $T=25^{\circ} \mathrm{C}$.

indicates deviation of less than $\pm 0.1 \%$. If we assume that the droplet rapidly equilibrates with the water vapor in the surrounding atmosphere, the relative humidity at the droplet can be calculated from the known water activity of sodium chloride solutions, ${ }^{25}$ as is illustrated by the black trace in Fig. 7(a). This analysis allows estimation of the RH standard deviation: $\sigma=0.03 \% \mathrm{RH}$.

\section{B. Aqueous solution thermodynamics}

Hydrophilic crystalline materials such as sodium chloride exhibit an asymmetric response to humidification (Fig. 8 ). The relative humidity controller was programed to expose a levitated sodium chloride particle to a triangular ramp between $40 \%$ and $78 \% \mathrm{RH}$ at a scan rate of $20 \% \mathrm{RH} / \mathrm{h}$. The temperature remained between 24.9 and $25.0{ }^{\circ} \mathrm{C}$ throughout the experiment. The vertical balancing voltage was continuously monitored by the position controller during electrostatic levitation. Relative mass was calculated by normalizing the instantaneous balancing voltage by the balancing voltage in the dry state. As the humidity increased, no water was absorbed until $75 \% \mathrm{RH}$, at which point the crystalline particle was spontaneously dissolved by uptake of water from the vapor to form a saturated aqueous solution droplet that continued to grow with further humidification. This transition is known as deliquescence. Reducing the humidity dried the droplet, increasing the solute concentration well above the saturation limit until, at approximately $50 \% \mathrm{RH}$, crystal nucleation initiated the sudden evaporation of all water and the particle returned to the dry state. This transition is known as efflorescence.

Pseudoequilibrium conditions apply during a sufficiently slow continuous scan. This can be verified by comparing the droplet mass as a function of $\mathrm{RH}$, with observations made when the RH is held constant sufficiently long to ensure that equilibrium is achieved. Measurements were made in discrete 30 min steps from $55 \%$ to $80 \%$ RH (Fig. 9). The solu- 


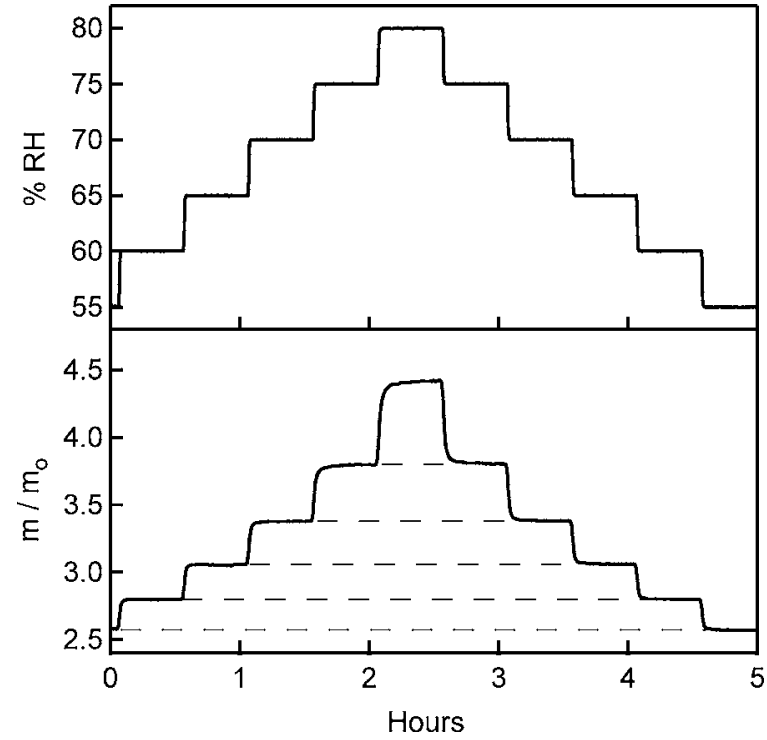

FIG. 9. Response of a supersaturated aqueous sodium chloride droplet to relative humidity steps. Particle mass was normalized by the mass of the dry state. $T=25^{\circ} \mathrm{C}$.

tion concentration equilibrates rapidly compared to the step time; increasing and decreasing steps yield identical results.

The droplet mass measured with a $20 \% \mathrm{RH} / \mathrm{h}$ scan agrees well with both the stepping mode data and a correlation previously developed by Tang et $_{\text {al. }}{ }^{25}$ based on stepping mode electrodynamic balance experiments (Fig. 10). Repeated scans demonstrate the reproducibility of the thermodynamic (water activity) measurements. Small variations appear in the deliquescence $\mathrm{RH}\left(\mathrm{RH}_{d}\right)$; larger variations appear in the efflorescence $\mathrm{RH}\left(\mathrm{RH}_{e}\right)$. The latter deviations reflect the stochastic nature of crystal nucleation that we will explore below.

\section{Nucleation statistics}

Solute nucleation is a stochastic process driven by fluctuations in a metastable solution. Once a stable nucleus

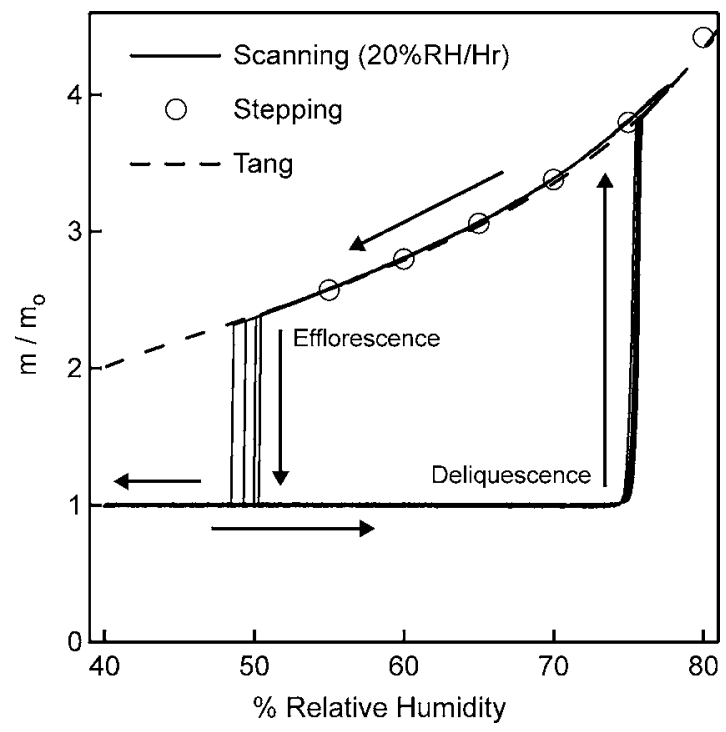

FIG. 10. Parametric plot of thermodynamic data for a sodium chloride particle. Current experiments were compared to a correlation previously published by Tang et al. (Ref. 25). $T=25^{\circ} \mathrm{C}$.

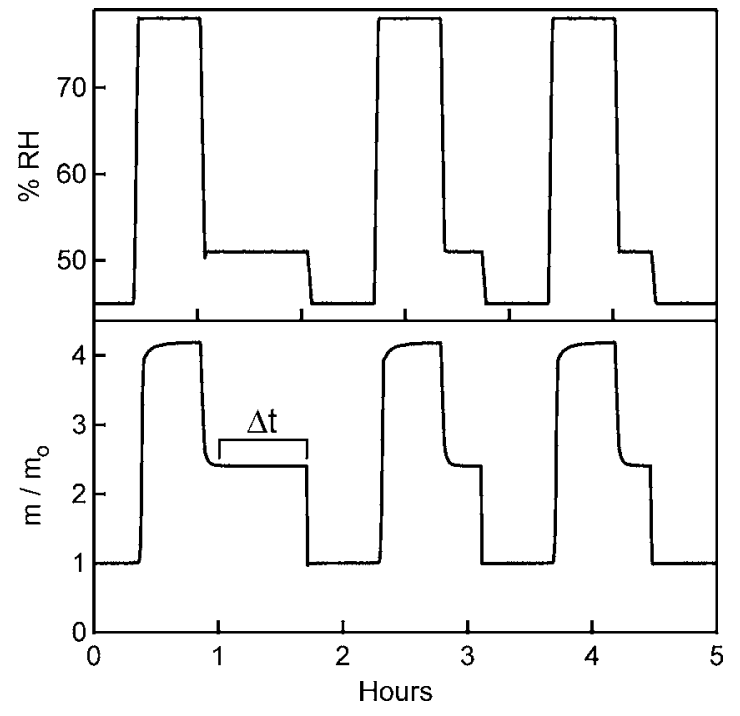

FIG. 11. Programed relative humidity history and particle response typical of the automated nucleation experiment.

forms, it rapidly grows, thereby producing an efflorescence event. The nucleation rate (number of events per unit volume per unit time) can be estimated as $J=\left(\langle\tau\rangle V_{p}\right)^{-1}$, where $\langle\tau\rangle$ is the average incubation time and $V_{d}$ is the droplet volume. Reliable estimation of the nucleation rate therefore requires many efflorescence event observations. The instrument described above enables accumulation of incubation time statistics through (i) automated detection of droplet efflorescence at a well-defined RH and (ii) subsequent processing to rapidly and accurately restore the supersaturated state (Fig. 11). A single sodium chloride particle was cycled unattended between a fully saturated state at $78 \% \mathrm{RH}$ and a supersaturated state at $51 \% \mathrm{RH}$ for $130 \mathrm{~h}$, during which time 78 nucleation events were observed. The temperature was maintained at $20.10 \pm 0.05{ }^{\circ} \mathrm{C}$ throughout the experiment. A dry crystalline sodium chloride particle deliquesces when the relative humidity is raised above $75 \%$. When the relative

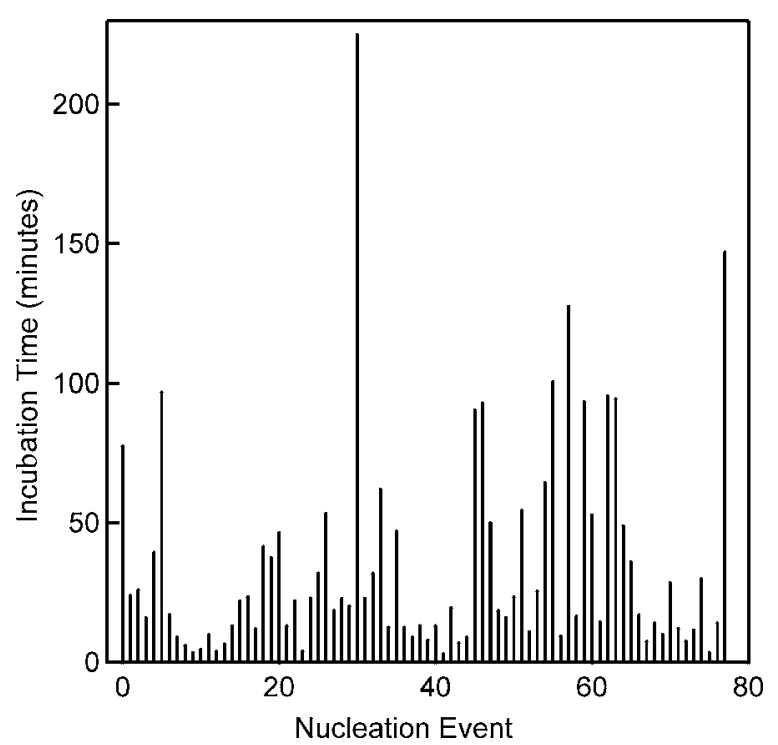

FIG. 12. Nucleation incubation times recorded during the nucleation experiment. The average recorded incubation time was $34 \mathrm{~min}$. 


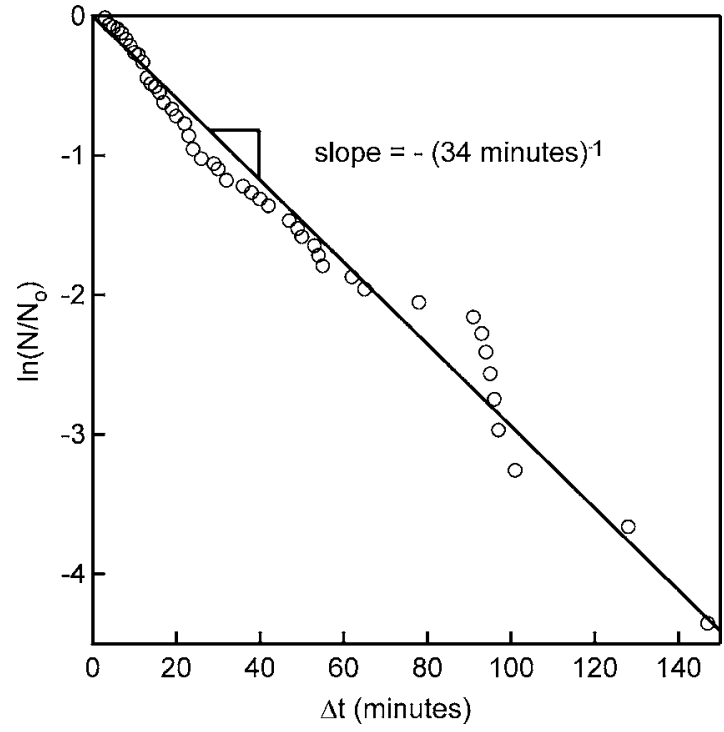

FIG. 13. Natural logarithm of the fraction of uncrystallized droplets remaining vs incubation time. The solid line represents exponential decay predicted by the average measured incubation time of $34 \mathrm{~min}$.

humidity is then reduced to $51 \%$, the solution droplet equilibrates to a supersaturated state with a relative mass of 2.4. The droplet remains in this metastable state until a nucleation event initiates crystallization of the entire particle accompanied by complete evaporation of water. Immediately after each nucleation event was detected the RH was further decreased to, and held briefly at, $45 \% \mathrm{RH}$ to thoroughly dry the particle and ensure a consistent starting point for the next cycle. While not essential for the present experiment, this additional drying is needed for materials that may retain water in the crystalline state, e.g., hydrophilic semicrystalline polymers that we are also probing with this apparatus. The abrupt reduction in mass to the dry state is automatically detected by software so that the cycle can be repeated. For each iteration the time between equilibration of the supersaturated state and nucleation is recorded as an incubation time.

Seventy-eight incubation times were compiled (Fig. 12) to determine an average measured incubation time of $34 \mathrm{~min}$, indicating that nuclei were formed at a rate of 4.9 $\times 10^{-4}$ nuclei/droplet/s. If the probability of observing a nucleation event is time independent, nucleation can be modeled as a Poisson process; crystallization of an ensemble of identical supersaturated droplets will then obey first-order kinetics. ${ }^{26}$ The ratio of uncrystallized droplets $(N)$ to the total number of droplets $\left(N_{o}\right)$ decays with time since the onset of supersaturation as predicted for a Poisson process, i.e., $\ln \left(N / N_{o}\right)=-t / \tau$ (Fig. 13). The close approximation to expo- nential decay gives no basis for rejecting the Poisson model.

The droplet diameter at $51 \% \mathrm{RH}$ is $33 \mu \mathrm{m}$ as determined from Mie scattering images. The corresponding droplet volume $\left(1.9 \times 10^{-8} \mathrm{~cm}^{3}\right)$ enables calculation of a volumetric nucleation rate of $2.6 \times 10^{4}$ nuclei $/ \mathrm{cm}^{3} / \mathrm{s}$. Given this measured rate and the experimental sample size of 78 , the actual nucleation rate is most likely bounded by $2.0 \times 10^{4}$ and 3.2 $\times 10^{4}$ nuclei $/ \mathrm{cm}^{3} / \mathrm{s}$, corresponding to a maximum uncertainty of $\pm 22 \%$ within the $95 \%$ confidence interval.

\section{ACKNOWLEDGMENTS}

This work was funded by the National Science Foundation (DMR-0505393 and the MRSEC Program under award DMR-0080065). The authors would also like to thank Professor Gary Lorden of Caltech for discussions regarding statistical analysis, and Professor Bill Bridges of Caltech for assistance in designing the optics for collection of Mie scattering.

${ }^{1}$ E. J. Davis, Aerosol Sci. Technol. 26, 212 (1997).

${ }^{2}$ C. B. Richardson and J. F. Spann, J. Aerosol Sci. 15, 563 (1984).

${ }^{3}$ I. N. Tang and H. R. Munkelwitz, J. Colloid Interface Sci. 98, 430 (1984).

${ }^{4}$ M. D. Cohen, R. C. Flagan, and J. H. Seinfeld, J. Phys. Chem. 91, 4563 (1987).

${ }^{5}$ Z. Liang and C. K. Chan, Aerosol Sci. Technol. 26, 255 (1997).

${ }^{6}$ S. Oatis, D. Imre, R. McGraw, and J. Xu, Geophys. Res. Lett. 25, 4469 (1998).

${ }^{7}$ J. M. Lightstone, T. B. Onasch, and D. Imre, J. Phys. Chem. A 104, 9337 (2000).

${ }^{8}$ D. Knezic, J. Zaccaro, and A. S. Myerson, J. Phys. Chem. B 108, 10672 (2004).

${ }^{9}$ M. A. Hamza, B. Berge, W. Mikosch, and E. Rühl, Phys. Chem. Chem. Phys. 6, 3484 (2004).

${ }^{10}$ B. Krämer, O. Hübner, H. Vortisch, L. Wöste, T. Leisner, M. Schwell, E. Rühl, and H. Baumgärtel, J. Chem. Phys. 111, 6521 (1999).

${ }^{11}$ R. A. Shaw and D. Lamb, Geophys. Res. Lett. 26, 1181 (1999).

${ }^{12}$ I. Weidinger, J. Klein, P. Stöckel, H. Baumgärtel, and T. Leisner, J. Phys. Chem. B 107, 3636 (2003).

${ }^{13}$ H. R. Pruppacher, J. Atmos. Sci. 52, 1924 (1995).

${ }^{14}$ H. Straubel, Z. Elektrochem. 60, 1033 (1956).

${ }^{15}$ R. F. Wuerker, H. Shelton, and R. V. Langmuir, J. Appl. Phys. 30, 342 (1959).

${ }^{16}$ W. H. Hartung and C. T. Avedisian, Proc. R. Soc. London, Ser. A 437, 237 (1992).

${ }^{17}$ S. Arnold, Rev. Sci. Instrum. 62, 3025 (1991).

${ }^{18}$ X. Zhang and E. Bar-Ziv, Rev. Sci. Instrum. 67, 3483 (1996).

${ }^{19}$ F. Zheng, X. Qu, and E. J. Davis, Rev. Sci. Instrum. 72, 3380 (2001).

${ }^{20}$ P. J. Wyatt and P. T. Phillips, J. Colloid Interface Sci. 39, 125 (1972).

${ }^{21}$ S. Arnold, J. Aerosol Sci. 10, 49 (1979).

${ }^{22}$ W. K. Rhim, S. K. Chung, D. Barber, K. F. Man, G. Gutt, A. Rulison, and R. E. Spjut, Rev. Sci. Instrum. 64, 2961 (1993).

${ }^{23}$ S. Schlemmer, J. Illemann, S. Wellert, and D. Gerlich, J. Appl. Phys. 90, 5410 (2001).

${ }^{24}$ H. C. van de Hulst, Light Scattering by Small Particles (Wiley, New York, 1957).

${ }^{25}$ I. N. Tang, A. C. Tridico, and K. H. Fung, J. Geophys. Res. 102, 23269 (1997).

${ }^{26}$ D. Turnbull, J. Chem. Phys. 20, 411 (1952). 